\title{
Orientation of Pterin-6-Carboxylic Acid on Gold Capped Silicon Nanopillars Platforms: Surface Enhanced Raman Spectroscopy and Density Functional Theory Studies
}

\author{
John J. Castillo, ${ }^{*, a}$ Ciro E. Rozo, ${ }^{b}$ Linda Bertel,,${ }^{c}$ Tomas Rindzevicius, ${ }^{d}$ \\ Stelia C. Mendez-Sanchez, ${ }^{a}$ Fernando Martínez, Ortega ${ }^{c}$ and Anja Boisen ${ }^{d}$ \\ ${ }^{a}$ Grupo de Investigación en Bioquímica y Microbiología, GIBIM, Escuela de Química, Universidad \\ Industrial de Santander, Bucaramanga, Colombia \\ ${ }^{b}$ Grupo de Investigaciones Ambientales para el Desarrollo Sostenible, Facultad de Química \\ Ambiental, Universidad Santo Tomas, Floridablanca, Colombia \\ ${ }^{c}$ Centro de Investigaciones en Catálisis, Universidad Industrial de Santander, Guatiguará, \\ UIS km 2 via Refugio, Piedecuesta, Colombia \\ ${ }^{d}$ Department of Micro and Nanotechnology, Technical University of Denmark, Lyngby, Denmark
}

\begin{abstract}
The orientation of pterin-6-carboxylic acid on gold nanopillars was investigated by surface enhanced Raman spectroscopy and density functional theory methods. The experimentally vibrations from pterin-6- $\mathrm{COOH}$ free and attached to the Au surface display vibration features indicating chemical interaction of the pterin with the metal surface. The spectral feature evidenced that the pterin would adsorb on gold surface with a "lying down" configuration through the high intensity vibration of $\mathrm{NH}$ scissoring and rocking $\mathrm{OH}$ modes. The orientation study of pterins on gold nanopillars presented herein is believed to lead to new applications in biosensing field for detecting pterins of physiological importance.
\end{abstract}

Keywords: SERS, gold nanopillar, pterin-6-carboxylic acid, DFT

\section{Introduction}

Surface enhanced Raman spectroscopy (SERS) are among the most widely spectroscopic techniques used for understanding the adsorption and orientation behavior of molecule on nanoscale metal surface. ${ }^{1-3}$ Furthermore, the fluorescence background displayed for some biomolecules is reduced when they are adsorbed on metal surfaces and, thus, the technique is useful in the study of biological samples. It is well known that SERS involve two mechanisms based on chemical and electromagnetic effects which allow a huge spectral enhancement as well as enough information of the adsorbed species.

Over the past decade, the interest for the synthesis and fabrication of new colloid metal nanoparticles and solid support based SERS substrates was increased and this has led to the detection at trace levels of different compounds. ${ }^{4}$ SERS substrates can be divided into two groups, $(i)$ colloid metal nanoparticles ${ }^{5}$ and (ii) roughened metallic surfaces. ${ }^{6}$

*e-mail: jcasleon@uis.edu.co
The main limitation in using colloid metal nanoparticles is the tendency for nanoparticle conglomeration after the addition of the analyte which often leads to poor reproducibility of the SERS spectra. ${ }^{7}$ An ideal SERS substrate must have sufficient Raman signal enhancement to be able to detect the analyte while, at the same time, having a homogenous surface able to produce reproducible Raman spectra. ${ }^{8}$ Various methodologies for fabrication of SERS substrates using e-beam lithography, ${ }^{9}$ anodized aluminum oxide templates ${ }^{10}$ and nanoimprinting ${ }^{11}$ have been reported. Recently, we have developed a new and simple maskless lithography method to produce flexible, free standing goldcapped nanopillar (Au NP) structures suitable for molecular detection at ultra-low concentrations. ${ }^{12,13}$

Numerous SERS experiments have been reported to study the orientation and adsorption mechanisms of molecules on SERS active substrates employing both quantum calculations and Raman scattering experiments. Wu et al. ${ }^{14}$ reported the chemical effects that arise between various molecules adsorbed on different metal clusters. An interesting work studied the effect of electron donators 
on para-substituted benzenethiols using SERS on gold nanoclusters. ${ }^{15}$ By employing SERS and electronic structure methods Geetha et al. ${ }^{16}$ studied the orientation of 1,4-dimethoxy-3-bromomethylanthracene-9,10-dione on silver nanoparticles.

SERS has been widely employed to elucidate information about the changes in the orientation of biomolecules due to the increase in the Raman scattering intensity by several orders of magnitude which are important to fabricate effective analytical tools. Stokes et al ${ }^{17}$ reported the detection of folic acid (FA) in urine samples using silver nanoparticles. In a similar approach a novel SERS binary substrate of graphene oxide was developed for ultrasensitive detection of FA. ${ }^{18}$

Pterins are a class of metabolites composed of a pteridine ring system with a keto group and an amino group on positions 4 and 2, respectively. Pterins are involved in a wide range of living systems and can participate in relevant biological functions. ${ }^{19}$ Detection of 5,6,7,8-tetrahydrobiopterin (BH4) in the urine of newborns is important for the study of folates deficiencies which can induce irreversible neurological damage. ${ }^{20}$ Pteridinic derivatives, such as pterin (PT) and pterin-6-carboxylic acid (Pt6C) are present in plasma, urine and other body fluids and the change in its normal concentrations is a valuable analytical signal for the diagnostics of various diseases.

Even though the SERS spectra of pterins have been investigated experimentally both on gold and silver nanoparticles, ${ }^{19,21}$ to the best of our knowledge there are no previous reports of the orientation studies and computed vibrational frequencies of pterins in metallic surfaces nanostructured.

In this study we examine the adsorption of Pt6C on gold metal nanostructures using SERS and density functional theory (DFT) calculations. In order to investigate the binding of Pt6C to nanostructured Au, SERS active Au NP substrates were utilized. The DFT method was employed to obtain vibrational spectra of free Pt6C and Pt6C bound to a cluster of ten gold atoms (Au10). The presented study is an explorative step towards the fabrication of a detection system that is capable of sensing deficiencies of pterins in body fluids of newborns.

\section{Experimental}

\section{Measurement tools and techniques}

Raman scattering and SERS measurements were performed using a Horiba scientific LabRam HR evolution (Yobim Yvon, USA) with an acquisition range of $50-4000 \mathrm{~cm}^{-1}$. A $785 \mathrm{~nm}$ laser excitation with powers of up to $25 \mathrm{~mW}$ and a diffraction grating of 600 lines $\mathrm{mm}^{-1}$ was used. Raman scattering spectrum of solid state Pt6C was collected using a $10 \times$ long working distance objective and the acquisition time was $30 \mathrm{~s}$ for 1 accumulation. 6PtC SERS spectra were recorded using a $10 \times$ long working distance objective, $0.1 \mathrm{~mW}$ laser power and $5 \mathrm{~s}$ signal accumulation times. The power density was kept ca. $12 \mathrm{~kW} \mathrm{~cm}^{-2}$ at the sample to minimize photoinduced and thermal effects. ${ }^{12}$

\section{Fabrication of SERS active Au NP substrates}

The substrates were fabricated employing reactive ion etching of Si and gold metal deposition processes yielding vertical free standing gold-capped silicon nanopillar structures (Au NP) surrounded by a continuous gold film at the base of the pillars. ${ }^{13,22}$ The obtained Au NP density is approximately 18 pillars $\mu^{-2}$. Au NP dimensions are the following: Au NP height ca. $600 \mathrm{~nm}$, Si pillar width ca. $50 \mathrm{~nm}$, Au cap height and width are ca. 300 and $120 \mathrm{~nm}$, respectively (see Supporting Information section). All fabricated Au NP structures were stored in a desiccator and utilized within 3 days to minimize any effects related to oxidation of the Au surface.

\section{Preparation of pterine-6-carboxylic acid solutions}

6PtC was obtained from Sigma-Aldrich Corp (USA). All chemicals used in this study were of analytical grade. Pt6C was dissolved in water with an addition of $250 \mu \mathrm{L}$ of $\mathrm{NaOH}\left(0.1 \mathrm{~mol} \mathrm{~L}^{-1}\right)$ due to the poor solubility of Pt6C. The Pt6C powder $(0.029 \mathrm{~g})$ was mixed with $\mathrm{H}_{2} \mathrm{O}(25 \mathrm{~mL})$ and magnetically stirring until the yellow color became clear.

\section{SERS measurements}

A droplet (ca. $1 \mu \mathrm{L}$ ) of a freshly prepared Pt6C solution was deposited on the SERS active substrates and left to dry. The droplets spread over the whole Au NP surface area $\left(5 \times 5 \mathrm{~mm}^{2}\right)$ in several minutes to ensure homogeneity in the surface coverage and to achieve a suitable time of adsorption equilibrium and thus avoid any change of the desorption of Pt6C. Before every SERS measurement Au NP-Pt6C were gently rinsed with Milli-Q water and dried with nitrogen flow to remove the non-adsorbed Pt6C. The Raman scattering signal was recorded over an area of ca. $0.05 \mathrm{~mm}^{2}$ with 17 and $11 \mu \mathrm{m}, \mathrm{x}$ and y step sizes, respectively.

\section{Computational methods}

In order to identify the main vibrations of Pt6Cs Raman scattering spectra its vibrational frequencies were 
calculated using DFT method with B3LYP functional2 ${ }^{23,24}$ combined with the standard $6-31 \mathrm{G}(2 \mathrm{~d}, \mathrm{p})^{25}$ basis set. The calculated frequencies were scaled by a factor of 0.9614 in accordance with previous results reported ${ }^{26}$ with the purpose to correct for systematic errors. A cluster of $\mathrm{Au}$ atoms was chosen as a model system for the Au NP structures. In order to decrease the high computational demands for larger clusters and to have a surface cluster with enough dimensions to interact with Pt6C, a cluster of $10 \mathrm{Au}$ atoms (Au10) was selected and proven to be a good candidate for simulating the Au-Pt6C SERS spectrum. The Au10 cluster was planar and large enough to cover a representative part of Pt6C, see the inset in Figure 1b. The interaction between Pt6C and Au10, the Au10-Pt6C geometry optimization and the Raman spectra were computed using the scalar relativistic effective core potential (ECP) with doublezeta basis sets (LANL2DZ). ${ }^{26}$ Validation of DFT for the calculation of gold cluster has been studied by Li et al. ${ }^{27}$ and DFT methods have been used successfully to optimize the structures and assign all vibrational bands and predict frequencies and energies of molecule-metal complexes..$^{28}$ The calculations were carried out using the GAUSSIAN 09 software package. ${ }^{29}$

\section{Results and Discussion}

\section{Raman spectra of Pt6C}

To the best of our knowledge the SERS spectra of pterins has been studied experimentally on gold and silver colloids nanoparticles and the literature has not discussed orientation, adsorption studies and computed vibrational frequencies of Pt6C in gold surfaces nanostructured. The assignment of Raman bands of pterins in a previously reported study was incomplete ${ }^{19,21}$ and a deeper analysis of all vibrational modes of Pt6C is warranted. In the following paragraph, the complete assignment of the Raman bands of Pt6C is presented using both experimental and theoretical spectra (see summarized data in Table 1). The optimized structure of the free Pt6C is depicted in the inset of Figure 1.

In Figure 1a calculated and experimental Raman scattering spectra of Pt6C are compared. The inset in Figure 1a shows the optimized structure of the Pt6C molecule. The calculated normal mode wavenumber of Pt6C in Figure 1a shows a set of intense peaks that can also observed experimentally (ca. 1600-1200 $\mathrm{cm}^{-1}$ ). The most intense experimentally observed bands are $\mathrm{N}-\mathrm{H}$ and $\mathrm{C}-\mathrm{H}$ rocking vibrations $(\rho(\mathrm{NH})(\mathrm{CH}))$ at $1526 \mathrm{~cm}^{-1}$ and $\mathrm{NH}_{2}$ wagging vibrations at 1311 and $1160 \mathrm{~cm}^{-1}$. These calculated wavenumbers are in agreement with the experimental wavenumbers recorded and in concordance with previous report about in situ detection of pterins on $\mathrm{Au}$ and $\mathrm{Ag}$ nanoparticles. ${ }^{21}$ A group of medium intensities frequencies located at 1567, 1372 and $1196 \mathrm{~cm}^{-1}$ are associated to $\rho(\mathrm{NH}), \rho(\mathrm{CH})$ and ring deformation from pterin moiety. A set of weak intensity bands at 787, 596 and $540 \mathrm{~cm}^{-1}$ are assigned to the wagging from $\mathrm{NH}_{2}$ group. A medium intensity band is observed at $947 \mathrm{~cm}^{-1}$ and is attributed to a ring deformation of Pt6C.
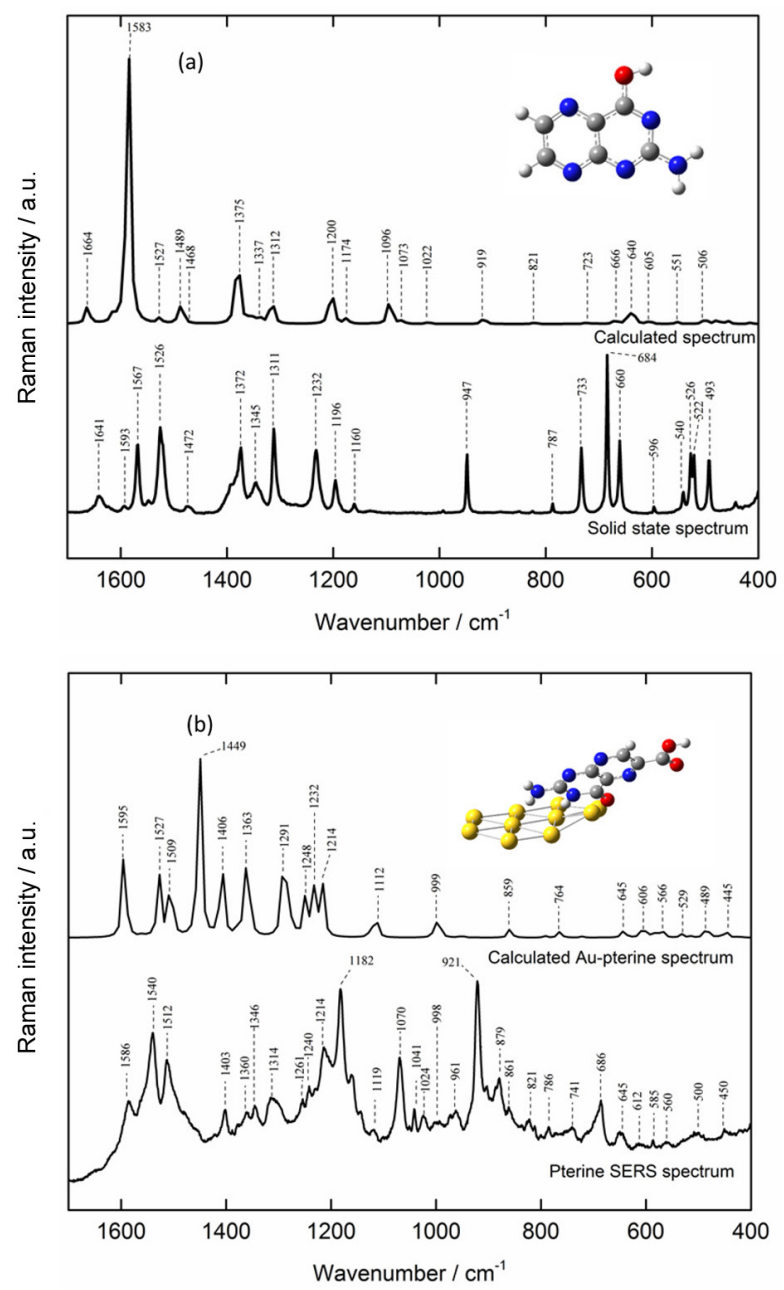

Figure 1. (a) DFT calculated and experimental Raman spectra of solid state Pt6C, the inset shows the optimized geometry for Pt6C; (b) DFT calculated and experimental SERS spectra of Pt6C using Au NP substrates. The inset shows optimized geometry for Pt6C adsorbed on a cluster of 10 gold atoms.

The calculated spectrum displays some deviations from the experimental results in the $1000-600 \mathrm{~cm}^{-1}$ region. A prominent example is the band found at $947 \mathrm{~cm}^{-1}$. The calculated spectrum displays a weak mode at $919 \mathrm{~cm}^{-1}$ which primarily derives from the ring deformation of the pterin.

\section{SERS spectra of Pt6C}

To have insight into orientation and adsorption behavior of Pt6C on gold capped silicon nanopillars the SERS 
Table 1. Assignment of experimental and calculated Raman modes of the pterine-6-carboxylic acid molecule (solid state)

\begin{tabular}{|c|c|c|}
\hline Raman $/ \mathrm{cm}^{-1}$ & Calculated $/ \mathrm{cm}^{-1}$ & Assignment \\
\hline $1641(\mathrm{~m})$ & 1664 & $\operatorname{sc}\left(\mathrm{NH}_{2}\right), \rho(\mathrm{NH})$ \\
\hline $1567(\mathrm{~m})$ & 1583 & $\rho(\mathrm{NH}) ; \mathrm{RD}$ \\
\hline $1526(\mathrm{~s})$ & 1527 & $\rho(\mathrm{NH})(\mathrm{CH}) ; \mathrm{RD}$ \\
\hline $1472(w)$ & 1489 & $\rho(\mathrm{NH})(\mathrm{CH})$ \\
\hline $1372(\mathrm{~m})$ & 1375 & $\rho(\mathrm{NH})(\mathrm{CH})(\mathrm{OH})$ \\
\hline $1345(w)$ & 1337 & $\rho(\mathrm{CH})(\mathrm{OH}), v(\mathrm{CO})$ \\
\hline $1311(\mathrm{~s})$ & 1312 & $\rho(\mathrm{NH})(\mathrm{CH}), \omega\left(\mathrm{NH}_{2}\right) ; \mathrm{RD}$ \\
\hline $1196(\mathrm{~m})$ & 1200 & $\rho(\mathrm{CH})(\mathrm{OH}) ; \mathrm{RD}$ \\
\hline \multirow[t]{2}{*}{$1160(w)$} & 1174 & $\rho(\mathrm{CH})(\mathrm{OH}), \omega\left(\mathrm{NH}_{2}\right)$ \\
\hline & 1096 & $\rho(\mathrm{OH})$ \\
\hline 947 (m) & 919 & $\mathrm{RD}$ \\
\hline $733(\mathrm{~m})$ & 723 & $\rho(\mathrm{OH}) ; \mathrm{RD}$ \\
\hline $660(\mathrm{~m})$ & 666 & $\rho\left(\mathrm{NH}_{2}\right)$ oop \\
\hline $645(\mathrm{~m})$ & 645 & $\rho\left(\mathrm{NH}_{2}\right)(\mathrm{OH})$ oop \\
\hline $596(w)$ & 605 & $\mathrm{RD}$ \\
\hline $540(w)$ & 551 & $\rho(\mathrm{CH})(\mathrm{OH})(\mathrm{CO})$ \\
\hline $493(w)$ & 506 & $\omega\left(\mathrm{NH}_{2}\right) ; b$ \\
\hline
\end{tabular}

w: weak; m: medium; s: strong; $v$ : stretching; $\delta$ : bending; $\rho$ : rocking; $\omega$ : wagging; sc: scissoring; t: twisting; b: breathing; oop: out of plane; $\mathrm{RD}$ : rings deformation of pterin.

spectrum of Pt6C is shown in Figure $1 \mathrm{~b}$ and the observed vibrational modes and its corresponding assignments are listed in Table 2. The assignment of the experimental SERS bands is based on the observed frequencies and intensity patterns of the experimental spectra of solid state Pt6C.

The presence of new bands and the relative intensification observed in SERS spectra compared to the normal Raman bands suggest an influence and close interaction of the atoms bound to the metal surface. The most intense set of bands present in Pt6C normal Raman spectrum are also found in its SERS spectrum, Figures 1a and 1b. Chemical interaction of the Pt6C molecule with the Au metal surface is evidenced by changes in both relative intensities and the position of the vibrational modes.

In Figure $1 \mathrm{~b}$ the strongest SERS signals are located at 1540 and $1512 \mathrm{~cm}^{-1}$ which arises mainly from scissoring from $\mathrm{NH}_{2}$ and ring deformation of pterin moiety. The SERS peak at $1586 \mathrm{~cm}^{-1}$ from the stretching vibration of $\mathrm{C}=\mathrm{O}(\mathrm{COOH})$ is a medium intensity band and it shows the interaction of carboxylic group with the Au surface. The weak SERS signal located around $1403 \mathrm{~cm}^{-1}$ show a multi-rocking vibration of $\mathrm{NH}$ and $\mathrm{CH}$ groups and is in a good agreement with the calculated spectra (see Figure 1b). The presence of the rocking vibrations out of the plane of the groups $\mathrm{NH}_{2}$ and $\mathrm{OH}$ at $645 \mathrm{~cm}^{-1}$ in the SERS spectrum
Table 2. Assignment of experimental and calculated Raman modes of the pterine-6-carboxylic acid molecule adsorbed on the Au surface

\begin{tabular}{|c|c|c|}
\hline $\begin{array}{l}\text { Surface enhanced } \\
\text { Raman spectroscopy } \\
\text { (SERS) / cm }{ }^{-1}\end{array}$ & Calculated $/ \mathrm{cm}^{-1}$ & Assignment \\
\hline $1586(\mathrm{~m})$ & 1595 & $v(\mathrm{C}=\mathrm{O}(\mathrm{COOH})), \rho(\mathrm{OH})$ \\
\hline $1540(\mathrm{~s})$ & 1527 & $\mathrm{sc}\left(\mathrm{NH}_{2}\right), \rho(\mathrm{NH})$ \\
\hline \multirow[t]{2}{*}{$1512(\mathrm{~s})$} & 1509 & $\rho(\mathrm{CH}) ; \mathrm{RD}$ \\
\hline & 1449 & $\rho(\mathrm{NH})(\mathrm{CH}) ; \mathrm{RD}$ \\
\hline $1403(\mathrm{~m})$ & 1406 & $\rho(\mathrm{NH})(\mathrm{CH})\left(\mathrm{NH}_{2}\right) ; \mathrm{RD}$ \\
\hline \multirow[t]{2}{*}{$1360(w)$} & 1363 & $\rho(\mathrm{NH}) ; \mathrm{RD}$ \\
\hline & 1291 & $\rho(\mathrm{CH})(\mathrm{OH}) ; \mathrm{RD}$ \\
\hline \multirow[t]{2}{*}{$1240(w)$} & 1248 & $\mathrm{RD}$ \\
\hline & 1232 & $\rho(\mathrm{OH})$ \\
\hline $1024(w)$ & 1214 & $\rho(\mathrm{OH})(\mathrm{CH})\left(\mathrm{NH}_{2}\right)$ \\
\hline $1112(w)$ & 1112 & $\rho\left(\mathrm{NH}_{2}\right) ; b$ \\
\hline $998(w)$ & 999 & $\rho\left(\mathrm{NH}_{2}\right)$ oop; RDoop \\
\hline $861(w)$ & 859 & $\rho(\mathrm{NH})$ oop \\
\hline $741(w)$ & 764 & $\rho(\mathrm{NH})(\mathrm{OH}) \mathrm{oop}$ \\
\hline $645(\mathrm{~m})$ & 645 & $\rho(\mathrm{CH})(\mathrm{OH})(\mathrm{CO})$ \\
\hline $612(w)$ & 606 & $\rho(\mathrm{OH}) ; b$ \\
\hline \multirow[t]{2}{*}{$585(w)$} & 566 & $\rho(\mathrm{NH})(\mathrm{OH}) ; b ; \mathrm{RD}$ \\
\hline & 529 & $\rho(\mathrm{NH})(\mathrm{OH}) ; b$ \\
\hline $500(w)$ & 489 & $\mathrm{RD} ; \mathrm{b}$ \\
\hline $450(w)$ & 445 & $\rho(\mathrm{NH})(\mathrm{OH}) ; \mathrm{RD}$ \\
\hline
\end{tabular}

w: weak; m: medium; s: strong; $v$ : stretching; $\delta$ : bending; $\rho$ : rocking; $\omega$ : wagging; sc: scissoring; t: twisting; b: breathing; oop: out of plane; $\mathrm{RD}$ : ring deformation of pterin.

further support the assumption that the Pt6C is anchored to the $\mathrm{Au}$ surface through a strong interaction with the amino group.

Such changes in the Pt6C SERS spectrum are corroborated by the calculated spectra obtained using the DFT method, Figure 2. Our theoretical study suggests an adsorption model where the Pt6C interacts with the $\mathrm{Au}$ atoms primarily through the nitrogen atoms from the amino group as shown in the inset in Figure $1 \mathrm{~b}$ and the model 4 in Figure 2. In order to further verify the reliability of the above model, we have simulated three additional geometries placing the Au10 cluster at different positions (see Figure 2, models 1, 2 and 3). The simulated SERS spectra from the models 1,2 and 3 shows a configuration where the amino group is far from the gold surface or the nitrogen from pterin moiety are interacting close together with gold atoms. On the other hand the models 1 and 4 showed the most strong vibration coming from the nitrogen from amino group and also the lowest values of energy, but the model 4 exhibited the lowest energy value 


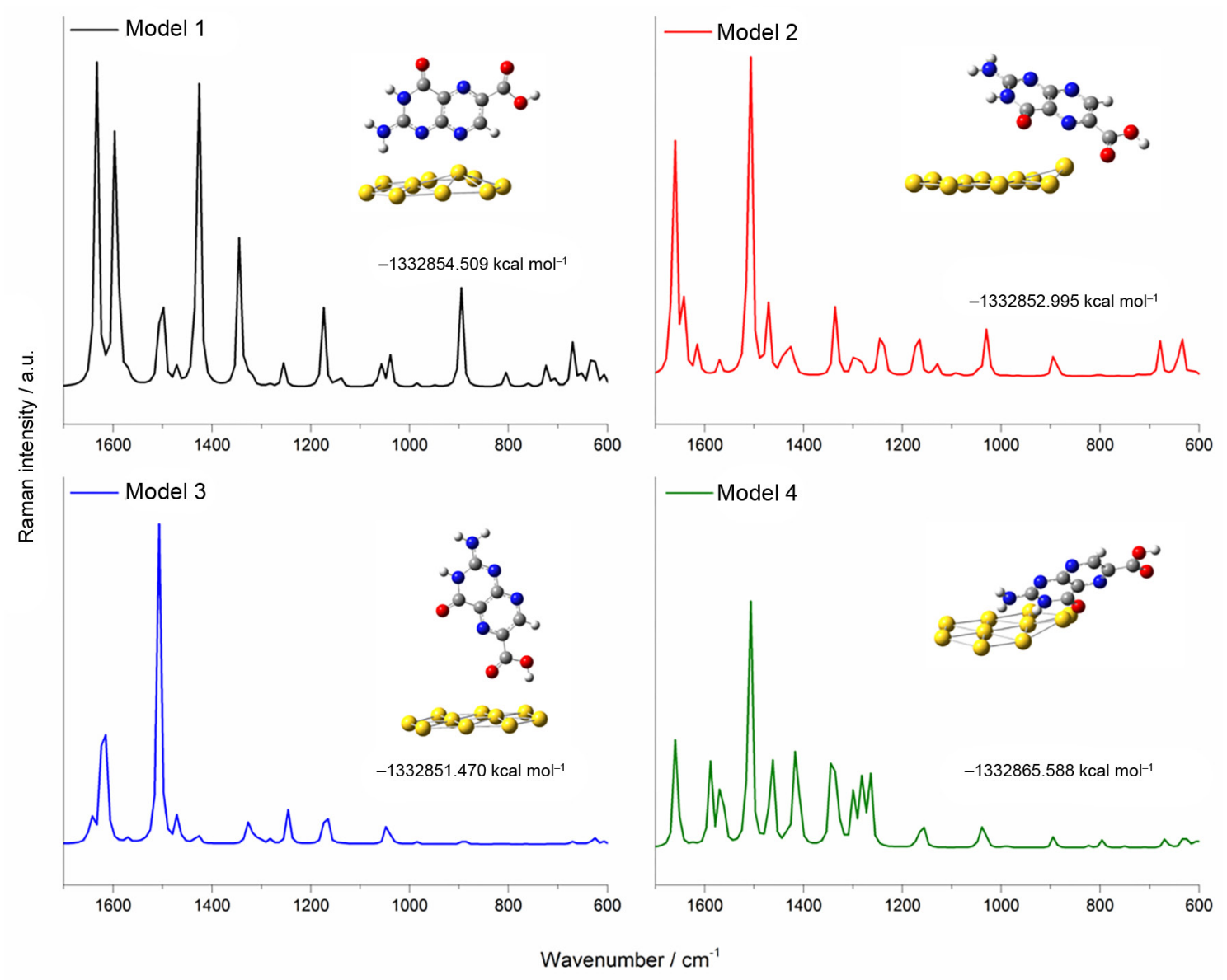

Figure 2. Raman spectra for different orientation geometries and energy values of Pt6C adsorbed on a 10 cluster of gold atoms.

$\left(\mathrm{E}_{4}=-1332865.588 \mathrm{kcal} \mathrm{mol}^{-1}\right)$ (inset Figure 2, model 4) confirming thus that the most likely interaction with the Au10 cluster is through the nitrogen of amino group.

After performing the optimization, we find no reliable geometry alternatives as the obtained energy values are higher than the optimized geometry shown in model 4 Figure 2.

The orientation of Pt6C on Au surface was determined on the basis of surface selection rules and it will depend on the active sites through which the interaction takes place. According to this rule, the vibrational mode with the transitional moment perpendicular to the surface should have a large enhancement in Raman peaks. The possible orientation of the Pt6C on the gold surface was lying down on the gold surface through bonding of the aromatic ring and the electron pair of the nitrogen in $\mathrm{NH}_{2}$ group of pterin. Geetha et al. ${ }^{16}$ observed a similar orientation behavior on an antraquinone derivate adsorbed on silver nanoparticles. The scissoring vibrations from $\mathrm{NH}_{2}$ and ring deformation of pterin are most prominent in SERS spectra of Pt6C confirming thus the lying down orientation of the pterin on the surface of gold capped silicon nanopillars.

\section{Frontier molecular orbitals}

Frontier molecular orbitals (FMOs) play important role in the chemical stability of the molecule. ${ }^{25}$ The highest occupied molecular orbital (HOMO) represents the ability to donate an electron while lowest unoccupied molecular orbital (LUMO) stands for an electron acceptor. The HOMO-LUMO energy gap determines a variety of properties including optical polarizability, kinetic stability and chemical hardness-softness of a molecule. ${ }^{26}$ The chemical hardness is usually a good indicator of the chemical stability. Hard molecules display a large HOMO-LUMO gap while soft molecules have a small HOMO-LUMO gap. The hardness of a molecule is defined as: $:^{25}$

$\eta=\frac{\left(-\varepsilon_{\text {HOMO }}+\varepsilon_{\text {LUMO }}\right)}{2}$

where $\varepsilon_{\text {номо }}$ and $\varepsilon_{\text {LUмо }}$ are the energies of the HOMO and LUMO orbitals, respectively.

The HOMO and LUMO energy was calculated using B3LYP/6-31G(d) method, see Figure 3. Results show that the HOMO is located over the all pterine molecule with a HOMO energy $(B 3 L Y P)=-0.24467$ a.u. 


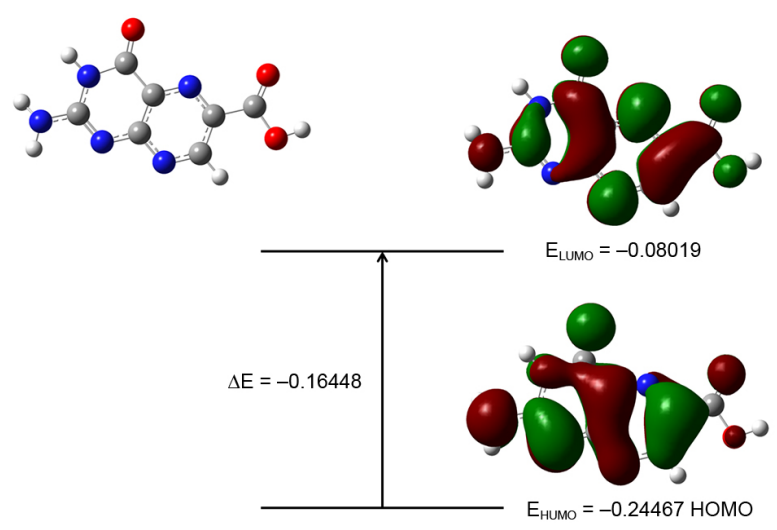

Figure 3. Calculated isodensity plots for the HOMO-LUMO energy levels of pterine-6-carboxylic acid.

while the LUMO is located in the pterin group except for some hydrogen atoms with a LUMO energy $(B 3 L Y P)=-0.08019$ a.u. Substituting the values into equation 1 yields $\eta=0.08224$ a.u.which indicates that Pt6C is a soft material. The chemical hardness of Pt6C is close to the physiological pterine FA whose activated form in body showed a reported value of $\eta=0.08175$ a.u. ${ }^{27}$

\section{Conclusions}

The study of the orientation of Pt6C on gold-capped silicon nanopillars is carried out using SERS spectroscopy and DFT calculations. The comparison between SERS and Raman scattering spectra show that the interaction of the Pt6C with the nanostructured gold surface is mainly through the nitrogen of the amino group and the orientation follows a lying down configuration confirmed by the bonding of the aromatic ring and the electron pair of the nitrogen in $\mathrm{NH}_{2}$ group of pterin. This feature was further confirmed by the calculation of the optimized geometry of Pt6C adsorbed to a cluster of ten gold atoms. HOMO and LUMO studies lead us to consider Pt6C as a soft material and chemical hardness value is close to the value exhibited by folic acid. The presented study is an explorative step towards the fabrication of a detection system that is capable of sensing pterins of physiological importance.

\section{Supplementary Information}

Supplementary information, including SEM image of the gold-capped silicon nanopillars, is available free of charge at http://jbcs.sbq.org.br as a PDF file.

\section{Acknowledgments}

We are grateful for the financial support provided by The Danish Concil for Independent Research's NAnoPLAsmonic
Sensors (NAPLAS) Sapere Aude project, Universidad Industrial de Santander (Project 1782) and Prof Rafael Cabanzo for helping us in Raman and SERS measurements.

\section{References}

1. Harper, M.; McKeating, K.; Faulds, K.; Phys. Chem. Chem. Phys. 2013, 15, 5312.

2. Howes, P.; Rana, S.; Stevens, M.; Chem. Soc. Rev. 2014, 43, 3835.

3. McNay, G.; Eustace, D.; Smith, W.; Faulds, K.; Graham, D.; Appl. Spectrosc. 2011, 65, 825.

4. Sonntag, M.; Klingsporn, J.; Zrimsek, A.; Sharma, B.; Ruvuna, L.; Van Duyne, R.; Chem. Soc. Rev. 2014, 43, 1230.

5. Muniz-Miranda, M.; Pergolese, B.; Muniz-Miranda, F.; Caporali, S.; J. Alloys Compd. 2014, 615, S357.

6. Wu, Q.; Luo, C.; Yu, H.; Kong, G.; Hu, J.; Chem. Phys. Lett. 2014, 608, 35.

7. Jarvis, R.; Johnson, H.; Olembe, E.; Panneerselvam, A.; Malik, M.; Afzaal, M.; O’Brien, P.; Goodacre, R.; Analyst 2008, $133,1449$.

8. Sharma, B.; Cardinal, M.; Kleinman, S.; Greeneltch, N.; Frontiera, R.; Blaber, M.; Schatz, G.; van Duyne, R.; MRS Bull. 2013, 38, 615.

9. Abu Hatab, N. A.; Oran, J. M.; Sepaniak, M. J.; ACS Nano 2008, 2, 377.

10. Wong-ek, K.; Eiamchai, P.; Horprathum, M.; Patthanasetthakul, V.; Limnonthakul, P.; Chindaudom, P.; Nuntawong, N.; Thin Solid Films 2010, 518, 7128.

11. Kondo, T.; Nishio, K.; Masuda, H.; Appl. Phys. Express 2013, 6, 102401.

12. Schmidt, M.; Hübner, J.; Boisen, A.; Adv. Mater. 2012, $24,11$.

13. Wu, K.; Rindzevicius, T.; Schmidt, S.; Mogensen, M.; Hakonen, K.; Boisen, A.; J. Phys. Chem. C 2015, 119, 2053.

14. Wu, D.; Liu, X.; Duan, S.; Xu, X.; Ren, B.; Lin, S.; Tian, Z.; J. Phys. Chem. C 2008, 112, 4195.

15. You, T.; Liang, X.; Gao, Y.; Yin, P.; Guo, L.; Yang, S.; Spectrochim. Acta, Part A 2015, 152, 278.

16. Geetha, K.; Umadevi, M.; Sathe, G.; Vanelle, P.; Terme, T.; Khoumeri, O.; J. Mol. Struct. 2014, 1059, 87.

17. Stokes, R.; McBride, E.; Wilson, C.; Girkin, J.; Smith, E.; Graham, D.; Appl. Spectrosc. 2008, 62, 371.

18. Hu, C.; Liu, Y.; Qin, J.; Nie, G.; Lei, B.; Xiao, Y.; Zheng, M.; Rong, J.; ACS Appl. Mater. Interfaces 2013, 5, 4760.

19. Smyth, C.; Mehigan, S.; Rakovich, Y.; Bell, S.; McCabe, E.; J. Biomed. Opt. 2011, 16, 77007.

20. Tornero, E.; Merás, I.; Espinosa-Mansilla, A.; Talanta 2014, 128,319 .

21. Stevenson, R.; Stokes, R.; MacMillan, D.; Armstrong, D.; Faulds, K.; Wadsworth, R.; Kunuthur, S.; Suckling, C.; Graham, D.; Analyst 2009, 134, 1561. 
22. Castillo, J.; Rindzevicius, T.; Wu, K.; Schmidt, M.; Janik, K.; Boisen, A.; Svendsen, W.; Rozlosnik, N.; Castillo-León, J.; J. Nanopart. Res. 2014, 16, 2525.

23. Rassolov, V.; Ratner, M.; Pople, J.; Redfern, P.; Curtiss, L.; J. Comput. Chem. 2001, 22, 976.

24. Lee, C.; Yang, W.; Parr, P.; Phys. Rev. B: Condens. Matter 1988, $37,785$.

25. Becke, A.; J. Chem. Phys. 1993, 98, 5648.

26. Pearson, G.; Proc. Natl. Acad. Sci. 1986, 83, 8440.
27. Li, L.; Cai, T.; Wang, Z.; Zhai, Z.; Geng, Y.; Sun, T.; Spectrochim. Acta Part A 2014, 120, 106.

28. Shi, Y.; Li, Z.; Fan, K.; J. Phys. Chem. A 2010, 114, 10297.

29. Lang, X.; Yin, P.; You, T.; Jiang, L.; Guo, L.; Chem. Phys. Chem. 2011, 12, 2468.

Submitted: October 8, 2015 Published online: December 15, 2015 